\title{
Mudança dos elementos meteorológicos em função da degradação da floresta urbana
}

\author{
Changes in meteorological elements due to the degradation of the urban forest
}

\author{
Henderson Silva Wanderley, Vanessa Claudino Miguel ${ }^{\mathrm{II}}$
}

\begin{abstract}
Resumo
O estudo das interações biosfera-atmosfera permite descrever e caracterizar os principais processos de transferência de energia e massa, relacionados às particularidades da vegetação. Assim, as interações que ocorrem em uma floresta podem sofrer impactos significativos em virtude de mudanças do clima ou no uso do solo. Deste modo, o objetivo desta pesquisa foi identificar o papel da degradação da floresta urbana da Tijuca na variabilidade de elementos meteorológicos. Para esse fim, foram realizadas medições em área de floresta conservada $\left(-22^{\circ} 95^{\prime} \mathrm{S},-43^{\circ} 28^{\prime} \mathrm{W}, 460 \mathrm{~m}\right)$ e degradada $\left(-22^{\circ} 95^{\prime} \mathrm{S},-43^{\circ} 27^{\prime} \mathrm{W}, 445 \mathrm{~m}\right)$, no Parque Nacional da Tijuca, localizado no município do Rio de Janeiro-RJ, com a utilização de duas estações meteorológicas automáticas com coleta de dados dos dias 18/07 a 09/08/2017, com intervalos de medição de 30 min, com medição da variabilidade da temperatura do ar, umidade relativa do ar e precipitação. Os dados foram submetidos ao coeficiente de correlação de Pearson, aos testes de hipóteses teste t, $\mathrm{F}$ e Kolmogorov-Smirnov. Os resultados mostraram diferenças de temperatura média maior do que $1^{\circ} \mathrm{C}$ para a área degradada, mas em uma escala diária pode ser maior do que $7^{\circ} \mathrm{C}$. A diferença da umidade relativa média foi próximo de $10 \%$, sendo menor em floresta degradada. No entanto, em alguns períodos diários a umidade do ar foi $30 \%$ menor na área degradada. A precipitação mostrou uma diferença de próximo de $10 \%$ entre as áreas. Os testes de hipóteses aplicados mostraram diferenças estatísticas significativas para a temperatura e umidade. A degradação da floresta da Tijuca contribui para modificar do microclima local em função do aumento da temperatura do ar e redução da umidade relativa ar.
\end{abstract}

Palavras-chave: Temperatura do ar; Umidade relativa do ar; Floresta da Tijuca

\begin{abstract}
The study of biosphere-atmosphere interactions allows to describe and characterize the main processes of energy and mass transfer related to the particularities of the vegetation. Thus, interactions occurring in a forest can suffer significant impacts due to climate change and changes in land use. That way, the objective of this research was to identify the importance of the degradation of Tijuca forest in the variability of meteorological elements. For this purpose, measurements were made in preserved $\left(-22^{\circ} 95^{\prime} \mathrm{S},-43^{\circ} 27^{\prime} \mathrm{W}, 445\right.$ $\mathrm{m})$ and degraded $\left(-22^{\circ} 95^{\prime} \mathrm{S},-43^{\circ} 27^{\prime} \mathrm{W}, 445 \mathrm{~m}\right)$ forest area in Tijuca National Park, located in the city of Rio de Janeiro-RJ state. Two automatic meteorological stations were used, and the data collection was from $18^{\text {th }}$ July to $8^{\text {th }}$ September, in 2017, with 30-minute measurement intervals with measurement of the variability of the air temperature the air, relative humidity of the air and precipitation. The data was submitted to the Pearson correlation coefficient, and the test hypotheses t, F and Kolmogorov-Smirnov. The results showed average temperature differences greater than $1^{\circ} \mathrm{C}$ for the degraded area with a daily range greater than $7^{\circ} \mathrm{C}$. The mean relative humidity difference was close to $10 \%$, being lower in the degraded forest. However, in some daily periods, the relative humidity was $30 \%$ smaller in the degraded area. The precipitation showed a difference of close to $10 \%$ among the areas. The hypothesis tests applied show statistically significant differences showing that there is a significant effect on the meteorological elements analyzed with forest degradation. The degradation of the Tijuca forest contributes to the modification of the local microclimate due to the increase of the air temperature and the relative air humidity reduction.
\end{abstract}

Keywords: Air temperature; Relative humidity; Tijuca forest

Meteorologista, Dr., Professor do Departamento de Ciências Ambientais, Instituto de Floresta, Universidade Federal Rural do Rio de Janeiro, BR465, Km 7, CEP 23897-000, Seropédica (RJ), Brasil. henderson@ufrrj.br (ORCID: 0000-0002-4031-3509)

II Estudante de Engenharia Florestal, Instituto de Floresta, Universidade Federal Rural do Rio de Janeiro, BR-465, Km 7, CEP 23897-000, Seropédica (RJ), Brasil. vanessa_clm@hotmail.com (ORCID: 0000-0001-7492-8563) 


\section{Introdução}

O estudo das interações biosfera-atmosfera permite descrever e caracterizar os principais processos de transferências de energia e massa, relacionados às particularidades da vegetação, propriedades do solo e características do clima de uma região. Assim, as interações que ocorrem em uma floresta podem sofrer impactos em virtude de mudanças do clima e mudanças no uso do solo.

As modificações no clima por ações antrópicas, em sua maioria são resultados de alterações na composição atmosférica devido ao aumento dos gases do efeito estufa. Segundo o Painel Intergovernamental de Mudanças Climáticas (INTERGOVENMANTEL PANEL ON CLIMATE CHANGE, 2013) existe cerca de $90 \%$ de chance de que as atividades humanas, principalmente a queima de combustíveis fósseis, sejam a causa do aquecimento atmosférico global desde meados do século XX.

As mudanças no uso do solo geralmente são devido à derrubada da floresta nativa ou à substituição da mesma por áreas agrícolas ou de pastagem. Nas cidades, o processo de urbanização altera os componentes do balanço energético (GAGE; COOPER, 2017). As superfícies urbanas armazenam maior quantidade de radiação solar incidente, fazendo com que o fluxo de calor sensível seja maior do que o fluxo de calor latente. Esse armazenamento faz com que a temperatura do ar seja maior nas cidades (PHELAN et al., 2015).

A substituição de uma floresta conservada por qualquer outro tipo de cobertura vegetal no solo, solo nu ou prédios, geralmente contribui para alterar a distribuição temporal e espacial do balanço de radiação, temperatura do ar e do solo, umidade do ar, poluição do ar, mudança no regime pluviométrico, evaporativo, sequestro de carbono, dentre outros (XIE et al., 2015; WANG et al., 2016; BOTTALICO et al., 2017; HOWE et al., 2017; SHOJAEI et al., 2017).

A crescente substituição de áreas naturais de floresta por centros urbanos não só leva à mudança dos elementos meteorológicos como também a perda da biodiversidade e o aparecimento das ilhas de calor urbanos (TAVARES; PATO; MAGALHÃES, 2012; GREEN et al., 2017). As principais consequências dessas modificações estão ligadas ao aumento do consumo de energia, redução da velocidade do vento e da dispersão de poluentes, como também aumento de doenças respiratórias humanas (BUYANTUYEV; WU, 2010).

As florestas urbanas desempenham efeito benéfico para a redução da temperatura e mitigação do efeito de ilha de calor urbana, ajustando o clima urbano local e minimizando as mudanças microclimáticas futuras (KONG; YIN; JAMES; 2014). O efeito de resfriamento causado pela floresta urbana é inerente à sombra densa proporcionadas por galhos e folhas, os quais limitam o aquecimento devido à incidência direta de radiação solar e reduz a temperatura do meio mediante a transpiração da água através das folhas.

Assim, medições de elementos meteorológicos em áreas de florestas conservada e degradadas em regiões urbanas surgem como uma alternativa essencial para o entendimento das respostas observadas em uma localidade que passou por mudanças profundas com sua degradação, principalmente para as regiões urbanas em que o papel da floresta e essencial no microclima local.

Esse entendimento permite desenvolver estratégias que mitiguem os efeitos dessas mudanças nos sistemas floresta-cidade-atmosfera, principalmente com relação à compreensão da dinâmica do fluxo de calor e massa entre a superfície terrestre e a atmosfera, mudanças nos elementos meteorológicos e no microclima. Diante do exposto, o objetivo dessa pesquisa consistiu em entender o papel da floresta urbana da Tijuca na distribuição dos elementos meteorológicos mediante sua degradação. 


\section{Material e método}

\section{Caracterização da área de estudo}

As medições foram realizadas no Parque Nacional da Tijuca, localizado no município do Rio de Janeiro-Brasil. O parque possui extensão de 3.953 ha de Mata Atlântica, apresentando relevo montanhoso, com altitude variando de 80 a $1.021 \mathrm{~m}$, com clima tropical de altitude. A vegetação é classificada como Floresta Ombrófila Densa, com trecho em variados estádios sucessionais e porções degradadas cobertas por diferentes espécies heliófilas (COELHO NETTO, 2005).

O parque está dividido em quatro setores: Setor A - Floresta da Tijuca, Setor B - Serra da Carioca, Setor C - Pedra da Gávea e Pedra Bonita e Setor D - Pretos Forros/Covanca. O experimento foi realizado no Setor A do parque, com a colocação de duas estações meteorológicas automáticas, Davis - Vantage Pro2, com medição de temperatura do ar, umidade relativa do ar e precipitação pluviométrica dos dias 18/07 a 09/08/2016, com intervalos de medição de 30 minutos.

As estações meteorológicas foram colocadas em áreas distintas do Setor A do parque, sendo uma em área conservada e outra degradada. A área de floresta conservada teve por ponto de coleta de dados a fase Sul do Maciço da Tijuca (-22 $\left.{ }^{\circ} 95^{\prime} \mathrm{S},-43^{\circ} 28^{\prime} \mathrm{W}, 460 \mathrm{~m}\right)$, composto por uma densa floresta ombrófila, com altura das árvores em torno de $20 \mathrm{~m}$. A área degradada, também localizada na fase Sul $\left(-22^{\circ} 95^{\prime} \mathrm{S},-43^{\circ} 27^{\prime} \mathrm{W}, 445 \mathrm{~m}\right)$, caracteriza-se por solo nu e algumas poucas heliófilas.

\section{Análise dos dados}

A análise foi realizada com a comparação da variabilidade da temperatura do ar e umidade relativa do ar medidos em floresta conservada e degradada, calculando a análise estatística, como média, variância e desvio padrão. Os dados de chuva não foram analisados por não apresentarem distribuição continua durante o período de medição, mas apresentou diferença próxima de 10\% entre as áreas de medições.

Os dados de temperatura do ar e umidade relativa do ar foram submetidos ao coeficiente de correlação de Pearson, e aos testes de hipóteses teste t, F e Kolmogorov-Smirnov. O coeficiente de correlação de Pearson $(p)$ foi utilizado para quantificar o grau de associação entre os elementos meteorológicos medidos em floresta conservada e degradada (Eq. 1).

$$
\rho=\frac{\operatorname{cov}\left(\mathrm{x}_{1} \mathrm{x}_{2}\right)}{\sigma_{1} \sigma_{2}}
$$

Em que: $\mathrm{x}_{\mathrm{i}}=$ elemento meteorológico em análise; cov = covariância; $\mathrm{q}_{\mathrm{i}}=$ desvio padrão.

Para verificar se a correlação entre os elementos meteorológicos em análise foi significativa, a correlação foi submetida ao teste t de Student, com n-2 graus de liberdade, e nível de significância estatística $\alpha=0,05$. O valor de a indica o risco de concluir por uma correlação existente, quando na verdade não existe correlação. A hipótese de nulidade $\left(\mathrm{H}_{0}\right)$ adotada, consiste na ausência de correlação, ou seja, $p=0$, enquanto que a hipótese alternativa $\left(\mathrm{H}_{1}\right)$ é de que ela existe, definida por $p \neq 0$. A probabilidade da evidência contra a hipótese nula foi verificada pelo p-valor.

O p-valor representa a probabilidade dos valores encontrados serem representativos, tendo em vista que a hipótese nula fosse verdadeira. Quando os valores obtidos na análise resultarem em um p-valor menor ou igual ao valor de $\alpha$ especificado antecipadamente, rejeita-se a hipótese nula. Quando p-valor é maior do que $\alpha$, a hipótese nula não pode ser rejeitada. Assim, para $\mathrm{p}$-valor $\leq \alpha$, correlação é estatisticamente significativa, rejeita $\mathrm{H}_{0}$ e a correlação é diferente 
de 0 . Para p-valor $>\alpha$, correlação não é estatisticamente significativa, não se rejeita $\mathrm{H}_{0}$, e a correlação não é diferente de 0 .

O teste $t$ de Student foi utilizado para verificar a existência de diferenças entre a média dos elementos meteorológicos em análise (Eq. 2). A hipótese de nulidade $\left(\mathrm{H}_{0}\right)$ adotada é de que a diferença entre as médias é igual a 0 ; enquanto que a hipótese alternativa $\left(\mathrm{H}_{1}\right)$ estabelece que a diferença entre as médias é diferente de 0 .

$$
\mathrm{t} \frac{\left(\bar{x}_{1}-\bar{x}_{2}\right)-\left(\mu_{\bar{x}_{1}}-\mu_{\bar{x}_{2}}\right)}{\sqrt{S S_{x_{1}}-s s_{x_{2}}}}
$$

Em que: $x i=$ média; $\mu$ = valor da hipótese nula; $\mathrm{SS}_{\mathrm{i}}=$ variância; $\mathrm{n}=$ número da amostra.

O nível de significância adotado para o teste foi $\mathrm{a}=0,05$, com $\mathrm{n}_{1}+\mathrm{n}_{2}-2$ graus de liberdade. A probabilidade de aceitar ou rejeitar a hipótese de nulidade foi avaliada pela veracidade de sua probabilidade, a qual é definida pela razão crítica do p-valor. Se o p-valor calculado for maior do que o nível de significância $\alpha=0,05$, não se rejeitar a hipótese nula $\mathrm{H}_{0}$.

A hipótese de igualdade das variâncias entre as séries temporais em análise foi testada pelo teste $\mathrm{F}$ (Eq.3), com $\mathrm{n}_{1}=\left(\mathrm{n}_{\mathrm{x}}-1\right)$ e $\mathrm{n}_{2}=\left(\mathrm{n}_{\mathrm{y}}-1\right)$ graus de liberdades e nível de significância $\alpha=0,05$ probabilidade. O intervalo de confiança definido pela Eq.4.

$$
\begin{aligned}
& \mathrm{F}=\frac{S S_{x}^{2}}{S S_{y}^{2}} \\
& i c=f_{x} \frac{S S_{y}^{2}}{S S_{x}^{2}}<f_{y} \frac{S S_{y}^{2}}{S S_{x}^{2}}
\end{aligned}
$$

Em que: $\mathrm{F}=$ teste $\mathrm{F} ; \mathrm{IC}=$ intervalo de confiança.

O teste de Kolmogorov-Smirnov (K-S) é um teste estatístico não paramétrico o qual foi utilizado para identificar se a distribuição de duas amostras se diferem. A hipótese nula $\left(\mathrm{H}_{0}\right)$ adotada neste teste é de que a distribuição das amostras é extraída da mesma distribuição. A estatística do teste é a diferença máxima absoluta entre as duas funções empíricas de distribuição cumulativa (Eq. 5).

$$
\mathrm{D}=\max _{x}\left[\mathrm{~F}\left(x_{1}\right)-\mathrm{F}\left(x_{2}\right)\right]
$$

Em que: $\mathrm{D}$ = teste K-S; F = são funções de distribuição empíricas das amostras.

A hipótese de nulidade é rejeitada se $\mathrm{D}$ calculado for maior do que o valor $\mathrm{D}$ críticos, calculado em função do número de amostras (n1, n2) (Eq. 6). Segundo Young (1977), à medida que o número de amostras aumenta, a diferença máxima entre as amostras fica menor.

$$
S_{(n)}=\sqrt{\frac{\left(n_{1}+n_{2}\right)}{n_{1} n_{2}}}
$$

Em que: $S(n)=$ valor crítico. 


\section{Resultados e discussão}

Para o período em análise, a temperatura do ar média na floresta conservada foi de $17,21^{\circ} \mathrm{C}$, enquanto que foi observado um aumento de $1,07^{\circ} \mathrm{C}$ na temperatura do ar média da floresta degradada (Tabela 1). Os resultados desta pesquisa ratificam os encontrados por Yin, Perry e Dixon (1989) e Santos et al. (2011) em fragmento de floresta urbana e regiões adjacentes com características urbanas, em que foram observadas temperaturas maiores do que $1^{\circ} \mathrm{C}$ nas regiões adjacentes à floresta.

\section{Tabela 1 - Estatística da temperatura do ar $\left({ }^{\circ} \mathrm{C}\right)$ medidos em floresta conservada e degradada.}

Table 1 - Air temperature $\left({ }^{\circ} \mathrm{C}\right)$ statistics measured in preserved and degraded forest.

\begin{tabular}{|c|c|c|c|c|c|}
\hline \multirow[b]{2}{*}{ Locais } & \multicolumn{5}{|c|}{ Temperatura $\left({ }^{\circ} \mathrm{C}\right)$} \\
\hline & $\begin{array}{l}\text { Máximo } \\
\text { valor }\end{array}$ & $\begin{array}{c}\text { Mínimo } \\
\text { valor }\end{array}$ & Média & $\begin{array}{l}\text { Desvio } \\
\text { padrão }\end{array}$ & Variância \\
\hline $\begin{array}{l}\text { Floresta } \\
\text { Conservada }\end{array}$ & 26,0 & 10,3 & 17,21 & 2,49 & 6,18 \\
\hline $\begin{array}{l}\text { Floresta } \\
\text { Degradada }\end{array}$ & 31,3 & 9,6 & 18,28 & 3,53 & 12,49 \\
\hline
\end{tabular}

Fonte: Autores (2019)

Os resultados obtidos nesta pesquisa indicaram que houve um efeito significativo no aumento da temperatura do ar em função da degradação da floresta. Resultados similares foram verificados por Yin, Perry e Dixon (1989), Makhelouf (2009), Petralli et al. (2014) e Shojaei et al. (2017).

Essa característica também foi observada nas temperaturas extremas máximas, cuja temperatura máxima na floresta degradada foi $5,30^{\circ} \mathrm{C}$ maior do que em área conservada. Resultado análogo ao apresentado por Yin, Perry e Dixon (1989) e Martini e Biondi (2015) para fragmento de floresta urbana e os demais ambientes estudados, os quais apresentaram diferença maior do que $5^{\circ} \mathrm{C}$ para as temperaturas máximas.

Essa diferença ocorre devido à interceptação da radiação solar pelas copas das árvores, fazendo com o que a resposta do aquecimento seja menor no interior da floresta. Os resultados obtidos mostram que a degradação da floresta pode impactar em um aumento significativo na temperatura da cidade do Rio de Janeiro.

A menor temperatura extrema mínima foi observada na área de floresta degradada, a qual apresentou temperatura de $9,60^{\circ} \mathrm{C}$, sendo $0,70^{\circ} \mathrm{C}$ menor do que na floresta conservada. Os resultados encontrados por Yin, Perry e Dixon (1989) e Galvão (2000) apresentaram-se análogos aos encontrados nesse estudo. No entanto, a diferença entre as temperaturas mínimas entre área de floresta e de pastagem foi maior.

O maior resfriamento noturno apresentado pelo solo sem cobertura é decorrente do resfriamento radiativo, resultado da perda de radiação de onda longa pela superfície sem cobertura. $\mathrm{Na}$ floresta, a presença de vegetação faz com que o efeito do resfriamento seja menos eficiente do que em áreas sem vegetação, em que foram observadas as menores temperaturas (Figura 1).

A variabilidade da temperatura do ar diária apresentou influência da precipitação em função da cobertura de nuvens, fazendo com que fosse observado redução da temperatura do ar. No período em análise foi observado $21,1 \mathrm{~mm}$ a área de floresta conservada e $23,4 \mathrm{~mm}$ em na floresta degradada; diferença em torno de $10 \%$ em função da proximidade entre as áreas em análises e da interceptação das chuvas pela copa das árvores. 


\section{Figura 1 - Variabilidade temporal da temperatura do ar e chuva medidos na floresta conservada e degradada.}

Figure 1 - Temporal variability of air temperature and rain measured in preserved and degraded forest.

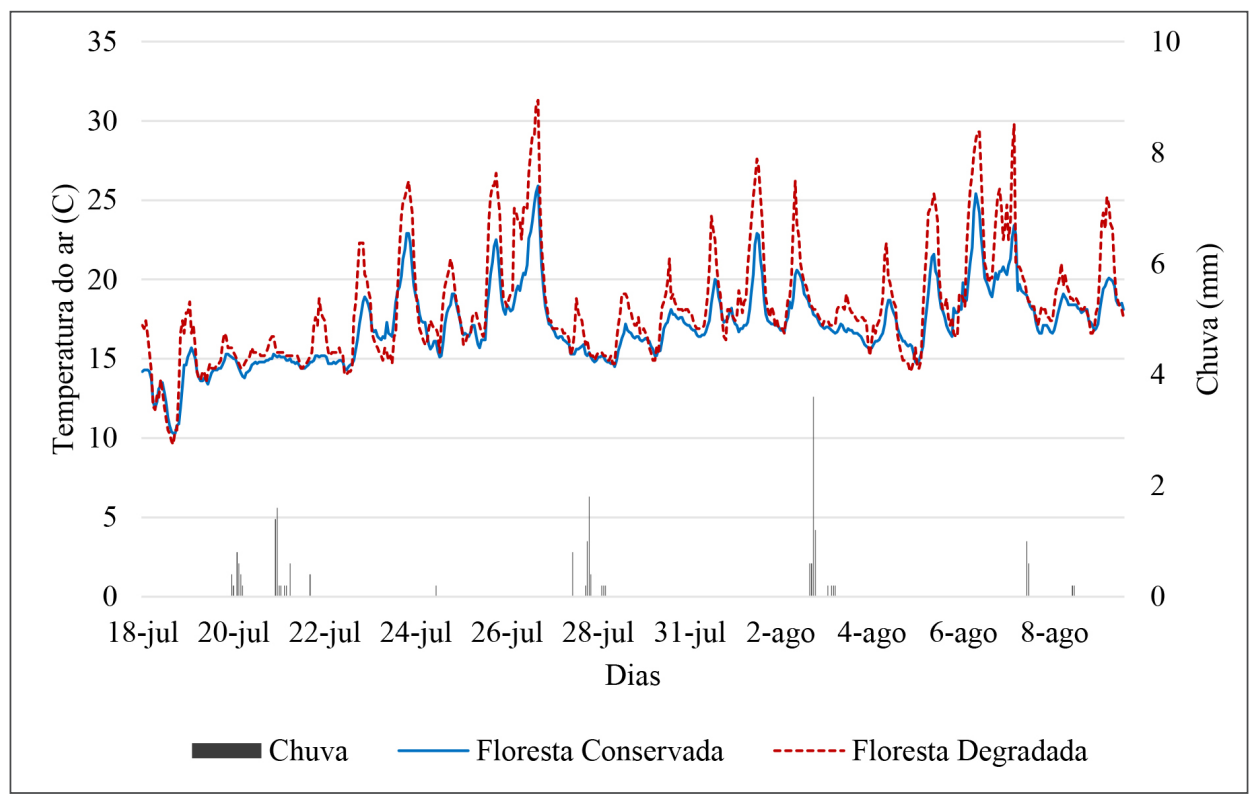

Fonte: Autores (2019)

O aquecimento diário observado entre a floresta conservada e degradada mostrou que, em mais de $60 \%$ dos dias analisados, a diferença entre a temperatura do ar medida em floresta conservada e degrada foi maior do que $4^{\circ} \mathrm{C}$. Destes dias, $71,5 \%$ apresentaram diferença entre as temperaturas do ar maior que $5^{\circ} \mathrm{C}$, chegando em um dia ser de $7,6^{\circ} \mathrm{C}$.

As maiores diferenças entre as temperaturas foram observadas no período da manhã, mas especificamente entre 9 e $11 \mathrm{~h}$, na maioria dos dias, o que também foi comprovado por Shojaei et al. (2017). Essa característica ocorre em função do aquecimento mais rápido do solo devido à ausência de cobertura vegetal, que por sua vez aquece o ar adjacente. Resultados semelhantes foram obtidos por Silva, Sá e Mota (2010) para floresta Amazônia.

Para a área de floresta, as máximas temperaturas diárias foram medidas entre 13 e 15 h $30 \mathrm{~min}$, com a maior frequência no intervalo de $13 \mathrm{~h} 30 \mathrm{~min}$ até $14 \mathrm{~h}$. Para a área degradada as máximas temperatura diárias foram medidas entre $11 \mathrm{~h} 30 \mathrm{~min}$ e $14 \mathrm{~h}$, com maior frequência coincidindo com os da floresta conservada. No entanto, foi observado um atraso de $30 \mathrm{mim}$ até $1 \mathrm{~h} 30$ min entre o aquecimento máximo observado em floresta conservada em relação à floresta degradada.

As principais características observadas na temperatura do ar medidas em floresta conservada e degradadas fizeram com que o desvio padrão e a variância dos dados de temperatura em floresta degradada fossem maiores do que os observados na floresta conservada. Estatística semelhante foi encontrada por Gage e Cooper (2017) e Shojaei et al. (2017).

O maior desvio padrão demonstra que os valores da série temporal analisada possuem variação em relação à média, com a quantificação da dispersão mostrada pela maior variância. Esse resultado se dá em função da maior amplitude térmica apresentada em área degradada, na qual foram observadas as máximas e mínimas temperaturas. Deste modo, a variabilidade dos elementos meteorológicos em floresta degradada foi menos uniforme e estável do que na floresta conservada, que teve o desvio padrão e variância menor. 
A umidade relativa do ar média na floresta conservada apresentou valor de $92,38 \%$, enquanto que na floresta degradada a umidade foi $83,88 \%$, uma redução de $8,50 \%$ (Tabela 2). Para floresta urbana, Martini e Biondi (2015) encontram valores de umidade relativa $16 \%$ maior quando comparada com áreas adjacentes urbanas.

A variabilidade observada nesse estudo, na umidade relativa do ar, foi semelhante aos valores encontrados por Dantas et al. (2011) para floresta tropical, com valor médio de 88\%, oscilando entre máximo e mínimo de $97 \%$ e $77 \%$, respectivamente. Os resultados apresentados por Galvão (2000) foram análogos aos encontrados nesses estudos.

\section{Tabela 2 - Estatística da umidade relativa do ar (\%) medidos em floresta conservada e degradada.}

Table 2 - Statistics of relative air humidity (\%) measured in preserved and degraded forest.

\begin{tabular}{lccccc}
\hline \multicolumn{1}{c}{ Locais } & $\begin{array}{c}\text { Máximo } \\
\text { valor }\end{array}$ & $\begin{array}{c}\text { Mínimo } \\
\text { valor }\end{array}$ & Média & $\begin{array}{c}\text { Desvio } \\
\text { padrão }\end{array}$ & Variância \\
\hline $\begin{array}{l}\text { Floresta } \\
\text { Conservada }\end{array}$ & 100 & 52 & 92,38 & 9,31 & 86,72 \\
$\begin{array}{l}\text { Floresta } \\
\text { Degradada }\end{array}$ & 99 & 28 & 83,88 & 16,55 & 273,96 \\
\hline
\end{tabular}

Fonte: Autores (2019)

Os valores máximos da umidade na floresta conservada apresentaram saturação ou valores muito próximos à saturação por um maior período do que na floresta degradada. $\mathrm{Na}$ área degradada, a umidade apresentou valores apenas próximos a saturação (Figura 2). A variação observada na umidade do ar apresentou diferença expressivas entre as áreas de medições no período em análise. Os períodos de saturação e umidade do ar elevada, em floresta conservada, ocorreram nos períodos posteriores as chuvas.

A maior umidade atmosférica observada na floresta conservada é resultado da interceptação e infiltração das chuvas, presença de nevoeiros, os quais são comuns na época do ano em análise, armazenamento de água nas copas e no solo. $\mathrm{Na}$ área degradada, a umidade proveniente para a atmosfera é decorrente apenas do solo.

Os valores de umidades mínimas foram os que apresentaram maior diferença entre as áreas em análise. Enquanto que o valor mínimo na floresta conservada foi de $52 \%$, na área de floresta degradada esse valor foi de $28 \%$. Durante o período em análise, em apenas 3 dias, em floresta conservada, foi observada umidade relativa do ar menor do que $60 \%$, cujos índices de umidade inferior a esse limiar causa desconforto para a saúde humana.

Em floresta degradada, o período observado de umidade relativa do ar menor do que $60 \%$ foi 3 vezes maior do que em floresta conservada. A variação diária mostrou redução da umidade do ar em área degrada no início da manhã, por volta das 7 h 30 min, com uma defasagem em torno de $30 \mathrm{mim}$ a $1 \mathrm{~h}$, para que ocorra a redução em floresta.

Essa redução se dá em torno de uma hora depois do nascer do sol, já que no período de medição foi realizado no inverno no Hemisfério Sul. Os menores valores de umidade diários em floresta degradada ocorreram entre $11 \mathrm{~h} 30 \mathrm{~min}$ e $14 \mathrm{~h}$. Para a floresta conservada foi observada a mesma defasagem do período na manhã entre os valores mínimos de umidade observados nas áreas de medição. A degradação da floresta conservada além de surtir impactos significativo na diminuição da umidade relativa do ar, pode impactar diretamente na formação e distribuição das chuvas na cidade do Rio de Janeiro. 


\section{Figura 2 - Variabilidade temporal da umidade relativa do ar e chuva medidos em floresta conservada e degradada.}

Figure 2 - Temporal variability of relative humidity measured and the rain in preserved and degraded forest.

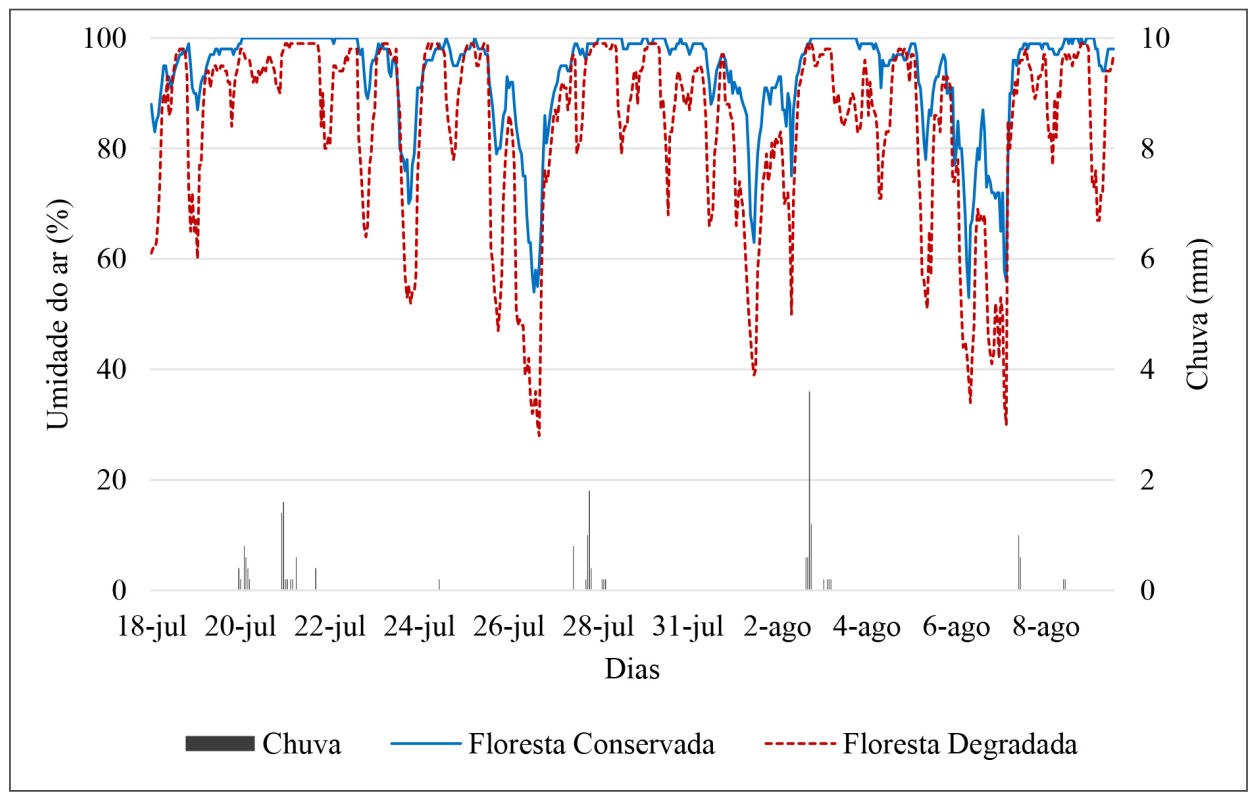

Fonte: Autores (2019)

Essa redução se dá em torno de uma hora depois do nascer do sol, já que no período de medição foi realizado no inverno no Hemisfério Sul. Os menores valores de umidade diários em floresta degradada ocorreram entre 11 h 30 min e 14 h. Para a floresta conservada foi observada a mesma defasagem do período na manhã entre os valores mínimos de umidade observados nas áreas de medição. A degradação da floresta conservada além de surtir impactos significativo na diminuição da umidade relativa do ar, pode impactar diretamente na formação e distribuição das chuvas na cidade do Rio de Janeiro.

A dispersão apresentada pela umidade em floresta degradada pode ser avaliada pelo desvio padrão e variância, nos quais se mostraram superiores na floresta degradada. Assim, pode ser ratificado a maior variabilidade da umidade relativa do ar em floresta degradada.

A razão da maior variabilidade observada na floresta degradada advém do grau de armazenamento de energia nas diferentes ocupações do solo entre as áreas, em que a floreta degradada apresenta solo nu, com alguma vegetação rasteira que permite receber maior fluxo de energia no solo, apresentando maiores oscilações diárias. Para a floresta conservada, a vegetação intercepta maior parte da energia solar, mantendo maior homogeneidade no interior do dossel.

A estatística aplicada para testar a hipótese de igualdade entre médias e variância para a temperatura do ar e umidade do ar, nas áreas em análises, mostrou p-valor menor do que o nível de significância adotado para todos os testes realizados. Deste modo, foi ratificado que a degradação de áreas de florestas conservada propiciou diferença estatística significativa entre a média e a variância da temperatura do ar e da umidade relativa do ar. A diferença estatística para variância também foi encontrada por Gage e Cooper (2017) e Howe et al. (2017).

Os resultados obtidos com a aplicação do teste estatístico de Kolmogorov-Smirnov ratificaram os resultados apresentados pelos demais testes realizados. Para esse teste, a hipótese de igualdade de distribuição dos elementos meteorológicos em análise, para as áreas de floresta conservada e degrada foi rejeitado. 
A correlação apresentada pela temperatura do ar entre a floresta conservada e floresta degradada mostrou correlação de $\mathrm{p}=0,9340$. Esse valor é superior ao encontrado por Howe et al. (2017). Os resultados do coeficiente de Pearson (p) para temperatura mostrou forte correlação entre as áreas analisadas, tendo sido adotada a classificação de Franzblau (1958). Para umidade relativa, a correlação demonstrada pelo coeficiente de Pearson foi satisfatória entre floresta conservada e degradada $(\mathrm{p}=0,8920)$, embora menor do que o obtido para a temperatura do ar devido à maior variabilidade de sua escala.

Os resultados obtidos pelo coeficiente de correlação mostram que a variabilidade da temperatura do ar entre as áreas em análise, assim como a da umidade relativa do ar, estão associadas e apresentam semelhanças na distribuição dos seus escores. O valor do coeficiente de correlação próximo de 1 , indicando maior grau de dependência estatística linear entre as variáveis em estudo. No entanto, a degradação da floresta fez com que a variabilidade dessas variáveis destoasse, principalmente nos períodos em que são medidos os extremos dessas variáveis, fazendo com que a correlação entre elas seja diferente de 1.

\section{Conclusões}

A degradação da floreta da Tijuca proporcionará impacto significativo para no microclima da floresta, e sobretudo na cidade do Rio de Janeiro, contribuindo para um aumento médio na temperatura do ar maior do que $1^{\circ} \mathrm{C}$, podendo ser maior que $7^{\circ} \mathrm{C}$ em uma escala diária.

A degradação da floreta da Tijuca induzirá uma redução média de $10 \%$ da umidade relativa do ar, tendo em vista que, em alguns períodos diários, a umidade do ar, em floresta degradada, foi $30 \%$ menor do que em floresta conservada.

\section{Agradecimentos}

Os autores agradecem ao Conselho Nacional de Desenvolvimento Científico e Tecnológico (CNPq), pelo apoio financeiro a esta pesquisa no financiamento do projeto 453876/2014-5. A Fundação de Amparo à Pesquisa do Estado do Rio de Janeiro (FAPERJ), pela concessão da bolsa de iniciação científica 200.697/2015, e ao Parque Nacional da Tijuca - Instituto Chico Mendes de Conservação da Biodiversidade (PNT/ICMBio).

\section{Referências}

BOTTALICO, F. et al. Spatially-explicit method to assess the dry deposition of air pollution by urban forests in the city of Florence, Italy. Urban Forestry \& Urban Greening, Amsterdam, v. 27, p. 221234, 2017.

BUYANTUYEV, A.; WU, J. Urban heat islands and landscape heterogeneity: linking spatiotemporal variations in surface temperatures to land-cover and socioeconomic patterns. Landscape Ecology, Dordrecht, v. 25, p. 17-33, 2010.

COELHO NETTO, A. L. A interface florestal-urbana e os desastres naturais relacionados à água no maciço da Tijuca: desafios ao planejamento urbano numa perspectiva sócio-ambiental. Revista do Departamento de Geografia, São Paulo, v. 16, p. 46-60, 2005.

DANTAS, V. A. et al. Fluxos de calor no dossel vegetativo e infiltração de água no solo, em floresta tropical. Revista Brasileira de engenharia agrícola e ambiental, Campina Grande, v. 15, p. 12661274, 2011.

FRANZBLAU, A. N. A primer of statistics for non statisticians. New York: Harcourt, Brace \& World, 1958. $150 \mathrm{p}$. 
GAGE, E. A.; COOPER D. J. Urban forest structure and land cover composition effects on land surface temperature in a semi-arid suburban area. Urban Forestry \& Urban Greening, Amsterdam, v. 28, p. 28-35, 2017.

GALVÃO, J. A. C. Balanço de energia em áreas de floresta e pastejem na Amazônia (Ji-Parana, RO). Revista Brasileira de Meteorologia, São José dos Campos, v. 15, p. 25-37, 2000.

GREEN, R. E. et al. A farming and the fate of wild nature. Science, Washington, v. 307, p. 550-555, 2005.

HOWE, D. A. et al. Spatial and temporal variability of air temperature across urban neighborhoods with varying amounts of tree canopy. Urban Forestry \& Urban Greening, Amsterdam, v. 27, p. 109116, 2017.

INTERGOVENMANTEL PANEL ON CLIMATE CHANGE. Climate Change. [S. l.]: The Physical Science Basis, 2013.

KONG, F.; YIN, H.; JAMES, P. Effects of spatial pattern of greenspace on urban cooling in a large metropolitan area of eastern China. Landscape and Urban Planning, Amsterdam, v. 128, p. 35-47, 2014.

MAKHELOUF, A. The effect of green spaces on urban climate and pollution. Iranian Journal of Environmental Health Science \& Engineering, [s. l.], v. 6, p. 35-40, 2009.

MARTINI, A.; BIONDI, D. Microclima e conforto térmico de um fragmento de floresta urbana em Curitiba, PR. Floresta e Ambiente, Seropédica, v. 22, p. 182-193, 2015.

PETRALLI, M. et al. Urban planning indicators: useful tools to measure the effect of urbanization and vegetation on summer air temperatures. International Journal of Climatology, Chinchester, v. 34, p. 1236-1244, 2014.

PHELAN, P. E. et al. Urban heat island: mechanisms, implications, and possible remedies. Annual Review of Environnment and Resources, [s. l.], v. 40, p. 285-307, 2015.

SANTOS, J. S. et al. Caracterização do campo térmico urbano e suas relações com o uso e ocupação do solo no Campus Central da UFPB. Revista Brasileira de Geografia Física, Recife, v. 3, p. 445-462, 2011.

SHOJAEI, P. et al. Effect of different land cover/use types on canopy layer air temperature in an urban area with a dry climate. Building and Environment, Amsterdam, v. 125, p. 451-463, 2017.

SILVA, L. M.; SÁ, L. D. A.; MOTA, M. U. S. Avaliação de características dos regimes de umidade na Flona de Caxiuanã-PA durante o experimento cobra-Pará. Revista Brasileira de Meteorologia, São José dos Campos, v. 25, p. 1-12, 2010.

TAVARES, A. O.; PATO, R. L.; MAGALHÃES, M. C. Spatial and temporal land use change and occupation over the last half century in a peri-urban area. Applied Geography, Amsterdam, v. 34, p. 432-444, 2012.

WANG, M. et al. Assessment of solar radiation reduction from urban forests on buildings along highway corridors in Sydney. Urban Forestry \& Urban Greening, Amsterdam, v. 15, p. 225-235, 2016.

XIE, J. et al. Environmental control over seasonal variation in carbon fluxes of an urban temperate forest ecosystem. Landscape and Urban Planning, Amsterdam, v. 142, p. 63-70, 2015.

YIN, X.; PERRY, J. A.; DIXON, R. K. Influence of canopy removal on oak forest floor decomposition. Canadian Journal of Forest Research, Ottawa, v. 19, p. 204-214, 1989.

YOUNG, I. T. Proof without prejudice: use of the kolmogorov-smirnov test for the analysis of histograms from flow systems and other. The Journal of Histochemistry and Cytochemistry, [s. l.] v. 25, p. 935-941, 1977. 